- Le groupage de certains éléments (circuits écourtés et groupés, surveillance, locaux laitiers, locaux pollués, ete.);

- Etc.

Ces trop courtes et trop peu nombreuses considérations montrent quelques-unes des interférences constructions-installations.

$$
*^{*} *
$$

Terminons en faisant la constatation qu'il s'agit là de données trop diverses pour être précisées en quelques mots.

C'est une conclusion à un texte qui aura peut-être montré l'extrême complexité du cas général de la construction et de l'installation d'usines de laiterie.

Le renouvellement constant de cette industrie, les travaux et efforts multiples des milieux participant à leurs études (services officiels et bureaux d'études, biologistes, sociétés d'équipements, fabricants de matériaux, constructeurs) ont cependant fait progresser très sensiblement en France, ces dernières années, l'art de construire et installer nos laiteries.

Ces travaux et efforts coordonnés vont se multiplier et font bien augurer de l'avenir.

\title{
LE LAIT DE BREBIS EN ISRAEL (1)
}

par

le $\mathrm{D}^{\mathrm{r}}$ A. KERN

\section{Généralités sur la situation laitière en Israel}

La situation générale en Israël en ce qui concerne le lait est dans les grandes lignes la suivante : la production annuelle totale de lait est d'environ 120 millions de litres dont 90 millions environ sont vendus. Pour une population de 1,5 million d'âmes, eeci eorrespond à moins de 70 litres par tête et par an. Cette quantité est très loin d'être suffisante pour répondre à la demande du lait de consommation, surtout pendant la saison chaude (été et automne) quand la production laitière tombe à environ $60 \%$ de la production annuelle moyenne. Pour augmenter la quantité de lait disponible, on est, par conséquent, obligé de distribuer comme lait de con-

(1) L'auteur de la présente étude a dirigé pendant de nombreuses années les plus importantes sociétés coopératives d'Israël et a publié récemment une brochure sur le . lait de brebis ».

Comme le lait de brebis n'a pas qu'un intérêt local, nous donnons ci-dessous une traduction des parties principales de cette brochure. (Traduction de O. Wolf.) 
sommation un lait de composition mixte consistant en un mélange de lait pur et de poudre de lait écrémé dissoute dans l'eau. La proportion de la solution ajoutée dépend de la quantité de lait pur disponible et s'élève pendant l'automne à $33 \%$.

Tout le lait de consommation est normalisé pendant toute l'année à $2,3 \%$ de matière grasse. Pour composer la matière grasse faisant défaut, la solution de poudre ajoutée se compose de $14 \%$ de lait écrémé en poudre et de $86 \%$ d'eau.

Tous les produits lactés (fromage de cottage mou; dérivés de laits acides et fromage demi-mou) sont uniquement préparés avec de la poudre de lait et des graisses végétales.

Le beurre n'est distribué qu'aux malades et aux enfants.

Il y a environ 90.000 bêtes de bétail en Israël, dont 40.000 vaches laitières. $80 \%$ du bétail appartient à une race de croisement Frisonne-Damscener, race qui s'est d'elle-même très bien adaptée au climat et donne des quantités considérables de lait, $10 \%$ du bétail consistent en une race importée d'Amérique pendant les toutes récentes années, le reste $(10 \%)$ étant représenté par des vaches de tous les pays du monde.

La quantité moyenne de lait produite annuellement par vache est de 3.400 kilogrammes, le maximum étant de 7.500 kilogrammes.

Sur la production totale de lait, environ 8 millions de litres sont du lait de brebis. Le dernier recensement a indiqué qu'il y avait en Israël environ 70.000 moutons, dont 50.000 brebis laitières. Les moutons appartiennent tous, presque sans exception, à la race Awassi, souche du mouton à grosse queue (Ovis platyura). Cette race est celle qui prédomine dans tous les pays arabes du MoyenOrient et en Turquie.

Environ 14.000 brebis laitières qui sont sous le contrôle permanent de l'association des éleveurs de mouton produisent en moyenne 240 kilogrammes de lait par an. Environ 6.000 brebis donnent 250 kilogrammes ot plus. La production maximum est de 680 kilogrammes de lait par an.

Outre le lait, les moutons donnent environ 100 tonnes de laine de qualité plutôt grossière et environ 500 tonnes de viande. Le fumier est très apprécié par une agriculture très extensive qui exploite de larges étendues de territoire du pays.

Pendant des dizaines d'années, l'élevage du mouton a été négligé par les fermiers d'Israël et n'a pu se développer que très lentement en dépit de ce que toutes les conditions nécessaires existaient: bons pâturages, exigences minimes en aliments fourragers, entretien facile (hangars), résistance aux maladies, et adaptation au climat.

C'est seulement au cours des récentes années qu'il est devenu 
clair que le développement de l'élevage du mouton est une besogne plus rapide et plus économique que l'élevage du bétail, puisqu'il faut moins d'argent étranger pour l'achat des moutons et de la nourriture supplémentaire. C'est pourquoi cette année de nombreux moutons seront importés de Turquie. D'autre part, le nombre des têtes de bétail n'augmentera que lentement en raison de ce que l'importation des vaches et l'alimentation coûteuse du bétail exigent de loin beaucoup plus d'argent étranger.

Les chèvres ne sont élevées en Israël que comme animaux domestiques. Comme ils nuisent aux exploitations forestières, il est interdit de faire pâturer les troupeaux de chèvres. Il y a environ 80.000 chèvres en Israël; mais il n'y a que très peu de lait de chèvre vendu.

\section{Composition du lait}

Les chiffres suivants relatifs à la composition des laits ressortent. des moyennes de plusieurs milliers de tests :

TABLEAU I

COMPOSITION DES LAITS DE VACHE, DE BREBIS ET DE GHẺVRE EN ISRAEL

\begin{tabular}{|c|c|c|c|}
\hline & $\begin{array}{c}\text { Lait de vache } \\
\text { (\%) }\end{array}$ & $\begin{array}{c}\text { Lait de brebis } \\
(\%)\end{array}$ & $\begin{array}{c}\text { Lait de ehèvre } \\
(\%)\end{array}$ \\
\hline Eau $\ldots \ldots \ldots \ldots \ldots$ & 87,5 & 81,6 & 86,8 \\
\hline Extrait sec $\ldots \ldots \ldots \ldots$ & 12,5 & 18,4 & 13,2 \\
\hline Matière grasse ........ & 3,7 & 7,5 & 4,3 \\
\hline Extrait sec dégraissé ... & 8,8 & 10,9 & 8,9 \\
\hline Protéines ............ & 3,2 & 5,6 & 3,8 \\
\hline Caséine $\ldots \ldots \ldots \ldots$ & 2,4 & 4,3 & 2,9 \\
\hline Lactose ............ & 4,9 & 4,4 & 4,3 \\
\hline \multirow[t]{3}{*}{ Substances minérales ... } & 0,7 & 0,9 & 0,8 \\
\hline & -二 & - - & 一一 \\
\hline & 100,0 & 100,0 & 100,0 \\
\hline
\end{tabular}

La composition du lait de vache se rapproche plutôt étroitement des chiffres donnés pour le bétail européen des Pays-Bas. La teneur en protéine et en caséine est faible. La teneur en matière grasse du lait de brebis est à peu près le double de celle du lait de vache, d'où une valeur élevée en calories; la teneur en caséine et en protéine est élevée ce qui est important pour la production du fromage et l'utilisation du sérum (fromage d'albumine). La teneur en lactose du lait de brebis ainsi que celle du lait de chèvre est remarquablement basse.

La période de vêlage pour les trois espèces tombe pendant les mois froids d'hiver. Le maximum de la production laitière a done 
lieu pendant les premiers mois de l'année, celle du lait de vache augmentant jusqu'en mai, puis diminuant rapidement ; le maximum pour la brebis est pendant février et mars.

Il y a quelques années, la livraison du lait de brebis était encore restreinte à la période de janvier à mai ; mais on a réussi récemment à prolonger cette période de décembre à juillet-août, ce qui, au point de vue économique, est de grande importance.

Il est spécialement intéressant de développer la teneur du lait de brebis et de vache en matière grasse. La teneur en matière grasse du lait de vache est en apparence indépendante de la période de lactation et suit très étroitement la courbe de la température extérieure. Pendant les mois froids, la teneur en matière grasse est élevée, diminuant (en même temps que la quantité de lait) pendant les mois chauds. Pendant la période de la plus forte consommation de lait dans le pays, c'est-à-dire pendant les derniers mois d'été, on ne peut avoir que de petites quantités de lait et son taux de matière grasse est faible, ceci étant en contradiction directe avec la théorie de l'augmentation du taux de matière grasse pendant la période de lactation. L'influence de la période de lactation peut être constatée dans le fait que la différence dans le pourcentage de matière grasse est relativement faible et qu'une plus grande chute pendant la forte chaleur de l'été est arrêtée.

Par contre, le climat n'a aucune influence sur la teneur en matière grasse du lait de brebis. De moins de $7 \%$ pendant le premier mois de lactation, la teneur en matière grasse augmente jusqu'à $9 \%$ à la fin de la période de lactation pendant le mois le plus chaud: août. Les deux tableaux qui suivent donnent les quantités de lait et le pourcentage de matière grasse au moment des livraisons aux laiteries de la TNUVA au cours de l'année $(70 \%$ de tout le lait vendu en Israël est traité par la TNUVA).

En ce qui concerne les fournitures de lait de brebis aux laiteries, nous avons à prendre en considération que souvent du lait de chèvre déclaré comme lait de brebis est fourni aux laiteries; mais ceci n'excède pas, toutefois, $15 \%$ de la quantité totale de lait. Malgré cette petite irrégularité, le tableau a été établi, car il donne un assez bon aspect des variations mensuelles des quantités et montre également clairement l'augmentation rapide de la quantité totale d'année en année. Les livraisons de cette année aux laiteries sont supérieures d'environ $25 \%$ à celles de l'année dernière. Le diagramme ci-dessous établi sur la base de la teneur en matière grasse du lait pur de brebis pendant une période de six ans illustre d'une façon intéressante les variations du taux de la matière grasse du lait de brebis. 


\section{TABLEAU II}

QUANTITÉ ET POURCENTAGE DE MATIÈRE GRASSE DU LAIT DE VACHE REGU PAR LES LAITERIES DE LA TNUVA

\begin{tabular}{|c|c|c|c|c|}
\hline \multirow[b]{2}{*}{ Mois } & \multicolumn{2}{|c|}{$1950-1951$} & \multicolumn{2}{|c|}{$1951-1952$} \\
\hline & $\begin{array}{c}\text { Matière } \\
\text { grasse (\%) }\end{array}$ & $\begin{array}{c}\text { Quantité } \\
\text { (en } 1.000 \mathrm{~kg} .)\end{array}$ & $\begin{array}{c}\text { Matière } \\
\text { grasse (\%) }\end{array}$ & $\begin{array}{c}\text { Quantité } \\
\text { (en } 1.000 \mathrm{~kg} .)\end{array}$ \\
\hline Octobre $\ldots \ldots \ldots \ldots \ldots$ & 3,71 & 2.372 & 3,67 & 2.832 \\
\hline Novembre .......... & 3,78 & 2.486 & 3,75 & 2.817 \\
\hline Décembre .......... & 3,85 & 2.682 & 3,77 & 3.180 \\
\hline Janvier $\ldots \ldots \ldots \ldots \ldots$ & 3,82 & 2.987 & 3,71 & 3.576 \\
\hline Février $\ldots \ldots \ldots \ldots \ldots$ & 3,82 & 2.873 & 3,69 & 3.664 \\
\hline 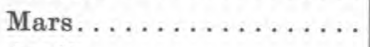 & 3,66 & 3. 767 & 3,59 & 4.477 \\
\hline Avril $\ldots \ldots \ldots \ldots \ldots$ & 3,51 & 3.822 & 3,47 & $4: 569$ \\
\hline Mai............... & 3,45 & 4.006 & 3,47 & 4.757 \\
\hline Juin $\ldots \ldots \ldots \ldots \ldots$ & 3,44 & 3.725 & 3,46 & 4.365 \\
\hline Juillet $\ldots \ldots \ldots \ldots \ldots \ldots$ & 3,43 & 3.429 & 3,49 & 4.264 \\
\hline Août $\ldots \ldots \ldots \ldots \ldots$. & 3,49 & 3.101 & 3,55 & 3.886 \\
\hline Septembre........... & 3,58 & 2.737 & 3,63 & 3.656 \\
\hline
\end{tabular}

TABLEAU III

QUANTITÉ ET POURGENTAGE DE MATIÉRE GRASSE DU LAIT DE BREBIS

\begin{tabular}{|c|c|c|c|c|c|c|}
\hline & \multirow{3}{*}{$\begin{array}{c}\text { Matière } \\
\text { grasse } \\
(\%) \\
1.740 \\
\text { échan- } \\
\text { tillons }\end{array}$} & \multirow{3}{*}{$\begin{array}{c}\text { Matière } \\
\text { grasse } \\
(\%) \\
\text { Trou- } \\
\text { peaux } \\
\text { sélec- } \\
\text { tionnés }\end{array}$} & \multicolumn{4}{|c|}{$\begin{array}{c}\text { Lait de brebis reçu par les laiteries } \\
\text { de la TNUVA }\end{array}$} \\
\hline & & & \multicolumn{2}{|c|}{$1950-1951$} & \multicolumn{2}{|c|}{$1951-1952$} \\
\hline & & & $\begin{array}{c}\text { Matière } \\
\text { grasse } \\
(\%)\end{array}$ & $\begin{array}{c}\text { Quantité } \\
(1.000 \mathrm{~kg} .)\end{array}$ & $\begin{array}{c}\text { Matière } \\
\text { grasse } \\
(\%)\end{array}$ & $\begin{array}{c}\text { Quantité } \\
(1.000 \mathrm{~kg} .)\end{array}$ \\
\hline Novembre........ & - & - & 7,35 & 1,5 & & \\
\hline Décembre ........ & - & 6,50 & 7,40 & 29,2 & 7,29 & 17,8 \\
\hline Janvier $\ldots \ldots \ldots \ldots$ & 7,51 & 7,42 & 7,52 & 167,2 & 6,88 & 141,3 \\
\hline Février $\ldots \ldots \ldots \ldots$ & 7,12 & 6,85 & 7,47 & 245,3 & 6,57 & 358,2 \\
\hline Mars $\ldots \ldots \ldots \ldots$ & 7,49 & 6,81 & 6,83 & 431,2 & 6,29 & 617,0 \\
\hline 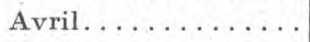 & 7,56 & 6,72 & 6,55 & 412,6 & 6,59 & 556,7 \\
\hline Mai $\ldots \ldots \ldots \ldots$ & 7,78 & 8,17 & 7,54 & 319,6 & 7,29 & 415,4 \\
\hline Juin $\ldots \ldots \ldots \ldots$ & 8,01 & 8,38 & 7,94 & 198,3 & 7,60 & 254,5 \\
\hline Juillet $\ldots \ldots \ldots \ldots$ & 8,09 & 8,63 & 8,05 & 98,2 & 7,83 & 132,3 \\
\hline Août $\ldots \ldots \ldots \ldots$ & 8,32 & 8,93 & 8,08 & 22,9 & 7,53 & 32,4 \\
\hline \multirow[t]{2}{*}{ Septembre. } & - & 10,04 & 8,58 & 0,4 & 7,33 & 1,4 \\
\hline & 7,51 & 7,57 & 7,25 & $1.927,4$ & 6,83 & $2.529,0$ \\
\hline
\end{tabular}




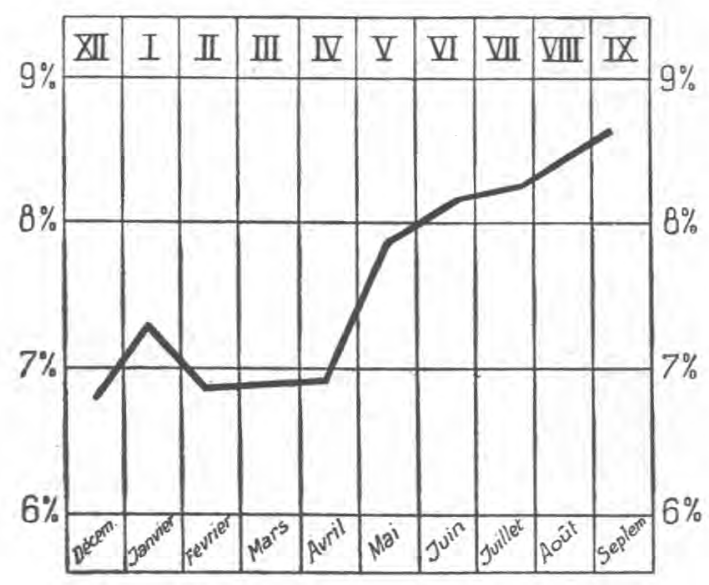

Diagramme :

Teneur du lait de brebis en matière grasse

La rapide augmentation de la teneur du lait de brebis en matière grasse entre avril et mai (environ $1 \%$ ) est spécialement remarquable. Il peut y avoir des raisons diverses à ce phénomène : le passage des hangars aux pâturages verts et en relation avec ce passage le changement complet d'alimentation, la fin de la période de vêlage, l'élévation considérable de la température extérieure, etc.

Pendant de longues années, le lait de brebis n'était pas accepté par les laiteries. Il était seulement utilisé dans les kibbutzim (colonies agricoles collectives) où on produisait d'une façon plutôt primitive un fromage de brebis dénommé Brinsen. Les laiteries donnaient diverses raisons à leur refus du lait de brebis : il était bien plus malpropre (macroscopiquement et microscopiquement), que le lait de vache, ce qui est encore exact! L'acidité du lait de brebis est très élevée; par suite de sa grande viscosité, il engorge les écrémeuses et les pasteurisateurs et, comme raison principale, il était surtout indiqué que sa teneur en matière grasse est si variable qu'il n'est pas possible de l'utiliser pour la préparation de produits normalisés. Je reviendrai ultérieurement à certaines de ces objections. Je veux d'abord donner les résultats d'un essai entrepris pour répondre à l'objection d'une teneur en matière grasse trop variable. Pendant une saison, 1.740 tests isolés furent effectués sur divers troupeaux. Ci-dessous le résultat et, à titre comparatif, je donne dans le même tableau les résultats de tests effectués par Richmond sur des laits de vaches.

En comparant ces chiffres, on voit qu'avec le lait de brebis, $93,4 \%$ des tests donnent une teneur en matière grasse entre 6,0 et $9,0 \%$ alors qu'avec le lait de vache $89,5 \%$ des tests donnaient une teneur en matière grasse entre 3,0 et $6,0 \%$. Si on tient compte de ce que la teneur moyenne en matière grasse d'un lait de brebis 
TABLEAUX IV et $\mathrm{V}$

FRÉQUENGE DU POURGENTAGE DE MATIÉRE GRASSE DANS LE LAIT DE BREBIS ET LE LAIT DE VAGHE

Lait de brebis (1.740 échantillons)

\begin{tabular}{|c|c|c|c|c|c|}
\hline \multirow[b]{2}{*}{ Plus de $10,0 \% \ldots$} & \multicolumn{2}{|r|}{$(\%)$} & \multirow[b]{2}{*}{ Plus de $8,0 \% \ldots$} & \multicolumn{2}{|r|}{$(\%)$} \\
\hline & 3 échantillons $=$ & 0,2 & & 31 échantillons $=$ & 0,5 \\
\hline De 9,0 à $10,0 \% \ldots$ & 54 échantillons $=$ & 3,1 & De 6,0 à $8,0 \% \ldots$ & 406 échantillons $=$ & 6,3 \\
\hline $\mathrm{De} 8,0$ \&े $9,0 \% \ldots$ & 411 échantillons $=$ & 23,6 & $\operatorname{De} 5,0$ à $6,0 \% \ldots$ & 1.103 échantillons $=$ & 17,1 \\
\hline De 7,0 à $8,0 \% \ldots$ & 837 échantillons $=$ & 48,1 & De 4,0 à $5,0 \% \ldots$ & 2,795 échantillons $=$ & 43,2 \\
\hline De 6,0 à $7,0 \% \ldots$ & 378 échantillons $=$ & 21,7 & De 3,0 à $4,0 \% \ldots$ & 1.886 échantillons $=$ & 29,2 \\
\hline De 5,0 à $6,0 \% \ldots$ & 51 échantillons $=$ & 2,9 & De 2,0 à $3,0 \% \ldots$ & 232 échantillons $=$ & 3,6 \\
\hline \multirow[t]{2}{*}{ Moins de $5,0 \% \ldots$} & 6 échantillons $=$ & 0,4 & Moins de 2,0\% $\quad$. & 9 échantillons $=$ & 0,1 \\
\hline & 1. 740 échantillons $=$ & 00,0 & & 6.492 échantillons $=$ & 100,0 \\
\hline
\end{tabular}

est approximativement deux fois plus élevée que celle du lait de vache, la conclusion doit être que le lait de brebis est bien plus stable dans sa teneur en matière grasse que le lait de vache. Alors que, ainsi qu'on le verra plus loin, la teneur en protéine et en caséine varie proportionnellement à la teneur en matière grasse, l'objection qu'il est difficile de normaliser les produits provenant du lait de brebis n'est pas soutenable. Ceci a été suffisamment prouvé par la pratique au cours de ces dernières années. Actuellement, le lait de brebis est accepté et traité par toutes les laiteries.

La teneur en matière grasse du lait de brebis dépend des intervalles entre les heures de traite.

Le fait bien connu, en ce qui concerne le lait de vache, que la teneur en matière grasse $d u$ lait dépend des intervalles entre les heures de traite se manifeste aussi avec le lait de brebis. Les brebis sont traites deux fois par jour, c'est-à-dire le matin de bonne heure et vers le milieu de la journée, de bonne heure l'après-midi (entre 12 et 16 heures suivant les circonstnaces).

Le tableau VI montre la différence existant entre la teneur en matière grasse et les quantités de lait obtenues entre la traite du matin et celle de l'après-midi. Nous pouvons donner les résultats obtenus sur deux animaux pendant une période de six mois.

\section{Traite principale et seconde traite}

La traite des brebis en Israël est effectuée de la façon suivante : le trayeur trait à grande vitesse la brebis attachée dans la bergerie sans chercher à effectuer une traite totale. Un second trayeur (ou dans les plus petits troupeaux le même trayeur) trait les ani- 


\section{TABLEAU VI}

EFFET DE L'INTERVALLE ENTRE LES TRAITES QUANTITÉ ET POURGENTAGE DE MATIÉRE GRASSE DU LAIT DU MATIN ET DU LAIT DE LAPRÉS-MIDI

\begin{tabular}{|c|c|c|c|c|c|c|}
\hline \multirow[b]{2}{*}{$\begin{array}{l}\text { Lait du matin ... } \\
\text { Lait de l'après-midi }\end{array}$} & \multicolumn{2}{|c|}{1 brebis pendant 6 mois } & \multicolumn{2}{|c|}{ I brebis pendant 6 mois } & \multicolumn{2}{|c|}{1 brebis pendant 1 jour } \\
\hline & $\begin{array}{l}6,41 \% \text { de } \mathrm{m} . \mathrm{g}: \\
9,17 \% \text { de } \mathrm{m} \cdot \mathrm{g} .\end{array}$ & $\begin{array}{r}126 \mathrm{~kg} . \\
75 \mathrm{~kg} . \\
\text { t } 1 \text { jour }\end{array}$ & $\begin{array}{l}5,61 \% \text { de m. g. } \\
7,48 \% \text { de m. g. }\end{array}$ & $\begin{array}{r}135 \mathrm{~kg} . \\
72 \mathrm{~kg} . \\
\text { brebis }\end{array}$ & $\begin{array}{l}4,60 \% \text { de m. g. } \\
9,80 \% \text { de m. g. } \\
\text { pendant } 1 \text { jour }\end{array}$ & $\begin{array}{r}1.000 \mathrm{gr} . \\
610 \mathrm{gr} .\end{array}$ \\
\hline Lait du matin .... & $10,5 \%$ de m. g. & $350 \mathrm{gr}$. & $\begin{array}{l}6,50 \% \text { de m. } g . \\
9,8 \% \text { de m. } g .\end{array}$ & $490 \mathrm{gr}$. & $\begin{array}{c}\text { poids spécifique } \\
1,0391 \\
\text { poids spécifique } \\
1,0347\end{array}$ & $\begin{array}{c}\text { extrait sec } \\
\text { dégraissé } \\
11,04 \\
\text { extrait sec } \\
\text { dégraissé } \\
10,62\end{array}$ \\
\hline
\end{tabular}

maux une seconde fois. La quantité obtenue dans la seconde traite est faible ; mais la teneur en matière grasse est très élevée.

Cette façon d'opérer a été adoptée après une longue pratique et on cherche à l'expliquer théoriquement en indiquant que les mamelles de la brebis sont différentes de celles des vaches et des chèvres; du reste, un trayeur expérimenté ne pourrait pas vider les mamelles en une seule traite. Le tableau suivant donne trois exemples de cette façon de traire.

TABLEAU VII

PREMIËE ET SEGONDE TRAITE : QUANTITÉ ET POURCENTAGe DE MATIËRE GRASSE

\begin{tabular}{|c|c|c|c|c|c|c|}
\hline & \multicolumn{2}{|c|}{$\begin{array}{c}\text { Traite du matin } \\
50 \text { brebis } \\
\end{array}$} & \multicolumn{2}{|c|}{$\begin{array}{l}\text { Traite du soir } \\
2 \text { brebis }\end{array}$} & \multicolumn{2}{|c|}{$\begin{array}{l}\text { Traite du matin } \\
11 \text { brebis }\end{array}$} \\
\hline & $\begin{array}{c}\text { Quantité } \\
\text { (kg.) }\end{array}$ & $\begin{array}{r}\text { Matière } \\
\text { grasse \% }\end{array}$ & $\begin{array}{c}\text { Quantité } \\
\text { (kg.) }\end{array}$ & $\mid \begin{array}{c}\text { matière } \\
\text { grasse } \%\end{array}$ & $\begin{array}{c}\text { Quantité } \\
\text { (kg.) }\end{array}$ & $\begin{array}{l}\text { matière } \\
\text { grasse } \%\end{array}$ \\
\hline Première traite ... & 34,5 & 6,75 & 1,85 & 7,20 & 9,3 & 5,62 \\
\hline Deuxième traite ..... & 5,8 & 9,90 & 0,22 & 10,40 & 0,8 & 9,73 \\
\hline
\end{tabular}

\section{Extrait sec et extrait sec dégraissé du lait de brebis}

Comme on peut s'y attendre étant données les variations de la teneur en matière grasse, l'extrait sec est aussi sujet à de fortes variations. L'extrait sec moyen s'élève à 18,4\%. On indique 15,4\% pour le minimum et $21,8 \%$ pour le maximum. L'extrait sec dégraissé est, toutefois, comme dans le lait de vache, un chiffre très stable. La 
valeur moyenne est $10,90 \%$ et les irrégularités de ce chiffre sont relativement faibles. Sur 1.740 tests de lait de brebis, 1.641, soit $94,3 \%$ donnent entre 10,4 et $11,4 \%$ ce qui signifie que l'irrégularité est de moins de $1 / 2 \%$. En ce qui concerne le lait de vache, on ne peut tirer que peu de conclusions sur sa composition et sa pureté de sa seule teneur en matière grasse. Il en est de même pour le lait de brebis. De même que lorsqu'on recherche une fraude du lait de vache l'extrait sec dégraissé est utilisé, il en est de même pour le lait de brebis. On suppose généralement avec raison que lorsque du lait de brebis a un extrait sec dégraissé inférieur à 9,8\% qu'il est fraudé avec de l'eau ou une autre sorte de lait. L'extrait sec dégraissé du lait de vache en-Israël est en moyenne de 8,76 , celui du lait de chèvre de $9,04 \%$.

Le tableau suivant donne la fréquence du pourcentage d'extrait sec dégraissé pour 1.740 tests de lait de brebis.

TABLEAU VIII

RÉPARTITION DU POURCENTAGE D'EXTRAIT SEG DÉGRAisSÉ (1.740 ÉCHANTILLONS)

$$
\begin{aligned}
& 2 \text { échantillons }=0,15 \\
& 32 \text { échantillons }=1,8 \\
& 833 \text { échantillons }=47,9 \\
& 808 \text { éehantillons }=46,4 \\
& 63 \text { échantillons }=\quad 3,6 \\
& 2 \text { échantillons }=0,15 \\
& 1.740 \text { échantillons }=100,0
\end{aligned}
$$

En dépit de cette stabilité élevée, de petites variations saisonnières (période de lactation) peuvent être notées et seront montrées sur trois exemples. La teneur en extrait sec dégraissé augmente d'après la teneur en matière grasse, mais diminue à la fin de la période de lactation. Néanmoins, dans l'ensemble, les variations sont très faibles.

\section{Protéine dans le lait de brebis}

Les analyses de trois sortes de protéines ont été effectuées : $1^{\circ}$ caséine $; 2^{\circ}$ protéine coagulée par la chaleur, appelée dans ce qui suit albumine et $3^{\circ}$ la protéine résiduaire, appelée globuline. Il ne nous paraît pas nécessaire d'insister davantage sur la valeur économique de la caséine. Après que la constatation de la forte teneur en albumine eut été établie, les laiteries procédèrent au chauffage et à l'acidification du sérum de lait de brebis obtenant ainsi jusqu'à 7 kilogrammes de fromage de sérum avec 100 kilogrammes de sérum. 
TABLEAU IX

VARIATIONS DE L'EXTRAIT SEG DEGRAISSÉ PENDANT LA SAISON

\begin{tabular}{|c|c|c|c|c|c|c|c|}
\hline T. & $\begin{array}{c}1.740 \\
\text { échantil } \\
\text { lons }(\%)\end{array}$ & \begin{tabular}{|c|} 
Tests dans \\
les laite- \\
ries (\%)
\end{tabular} & $\begin{array}{c}\text { Troupeaux } \\
\text { sélection- } \\
\text { nés (\%) }\end{array}$ & & $\begin{array}{c}1.740 \\
\text { échantil- } \\
\text { lons (\%) }\end{array}$ & \begin{tabular}{|c|} 
Tests dans \\
les laite- \\
ries (\%)
\end{tabular} & $\begin{array}{c}\text { Troupeaux } \\
\text { sélection- } \\
\text { nés (\%) }\end{array}$ \\
\hline Décembre & - & 10,41 & 10,36 & Mai ... & 10,80 & 11,01 & 11,03 \\
\hline Janvier .... & 11,19 & 10,51 & 10,43 & Juin . & 10,89 & 11,04 & 11,11 \\
\hline Février .... & 10,93 & 10,78 & 10,88 & Juillet ..... & 10,62 & 11,16 & 10,98 \\
\hline Mars ..... & 10,91 & 10,61 & 10,63 & Août ..... & 10,67 & 10,37 & 10,30 \\
\hline Avril ..... & 10,83 & 10,90 & 10,87 & Septembre & - & 10,55 & - \\
\hline
\end{tabular}

La teneur totale en protéine du lait de brebis s'élève en moyenne à $5,56 \%$, la teneur moyenne en caséine à $4,28 \%$, la teneur en albumine et globuline à $1,28 \%$ (les chiffres correspondants pour le lait de vache étant dans ce pays : teneur totale en protéine, $3,16 \%$; en caséine, $2,43 \%$; en albumine, $0,47 \%$; en globuline, $0,23 \%$ ). Une partie du lait écrémé de brebis est transformé en fromage mou et nous obtenons de 100 kilogrammes environ 24 kilogrammes de fromage ou approximativement 10 kilogrammes de plus que de 100 kilogrammes de lait écrémé de vache. Ceci suffit pour illustrer l'importance de la caséine. Il est remarquable qu'au début de la saison nous n'obtenons que 20 à 21 kilogrammes de fromage, tandis qu'en juillet-août le rendement va jusqu'à 27 kilogrammes. Ceci tient à la variation saisonnière de la teneur en caséine. Toutes les protéines sont sujettes à des variations ainsi que l'indique le tableau suivant :

TABLEAU $X$

\begin{tabular}{|c|c|c|c|c|c|}
\hline & \multirow{2}{*}{\begin{tabular}{|c|} 
Caséine sur \\
1.740 \\
échantil- \\
lons $(\%)$
\end{tabular}} & \multicolumn{2}{|c|}{ Essais dans les laiteries } & \multicolumn{2}{|c|}{ Troupeaux sélectionnés } \\
\hline & & $\begin{array}{c}\text { Protéine } \\
(\%)\end{array}$ & $\begin{array}{c}\text { Caséine } \\
(\%)\end{array}$ & $\begin{array}{c}\text { Protéine } \\
(\%)\end{array}$ & $\begin{array}{c}\text { Caséine } \\
(\%)\end{array}$ \\
\hline Décembre ........ & - & 5,20 & 3,86 & 4,97 & 3,81 \\
\hline Janvier .......... & 4,13 & 4,80 & 3,63 & 4,76 & 3,73 \\
\hline Février .......... & 4,16 & 5,30 & 4,21 & 5,24 & 4,27 \\
\hline $\operatorname{Mars} \ldots \ldots \ldots \ldots$ & 4,23 & 5,12 & 3,95 & 5,08 & 3,89 \\
\hline Avril $\ldots \ldots \ldots \ldots$ & 4,32 & 5,18 & 3,98 & 5,40 & 4,22 \\
\hline Mai $\ldots \ldots \ldots \ldots$ & 4,39 & 5,54 & 4,12 & 5,59 & 4,18 \\
\hline Juin $\ldots \ldots \ldots \ldots$ & 4,38 & 5,95 & 4,79 & 6,23 & 4,79 \\
\hline Juillet . . . . . . & 4,39 & 6,14 & 4,62 & 5,97 & 4,62 \\
\hline Août $\ldots \ldots \ldots \ldots$ & 4,76 & 6,18 & 4,37 & 6,18 & 4,89 \\
\hline Septembre ...... & - & 7,17 & 4,84 & - & \\
\hline
\end{tabular}


Il a été indiqué que la teneur en caséine la plus faible était de $3,63 \%$ et la teneur maximum de $4,89 \%$; mais très peu des analyses se sont écartées de $\pm 0,5 \%$ de la moyenne de $4,3 \%$. La littérature scientifique nous a appris qu'on a souvent essayé de déduire de la teneur en matière grasse une formule pour la teneur en caséine, ce qui a donné en fin de compte la formule suivante : caséine $-2 / 5^{\mathrm{e}}$ de matière grasse $+0,9$. A mon avis, cette formule donne des résultats trop bas pour le lait de vache à laquelle elle s'applique ; mais convient plutôt bien pour le lait de brebis.

Lactose et matières minérales (cendres). - La teneur en lactose du lait de brebis au début de la période de lactation est approximativement la même que celle du lait de vache. Toutefois, la chute de la teneur en lactose à la fin de la période de lactation est remarquable. Le tableau suivant donne un aperçu de l'évolution du lactose et des matières minérales dans le lait de brebis. Les matières minérales du lait de brebis dépassent de $0,3 \%$ celles du lait de vache. A la fin de la période de lactation, elles augmentent comme celles du lait de vache.

TABLEAU XI

TENEUR DU LAIT DE BREBIS EN LAGTOSE ET EN MATIERES MINÉRALES

\begin{tabular}{|c|c|c|c|c|c|}
\hline . & $\begin{array}{c}\text { Lactose } \\
(\%)\end{array}$ & $\begin{array}{l}\text { Matières } \\
\text { minérales } \\
(\%)\end{array}$ & & $\begin{array}{c}\text { Lactose } \\
(\%)\end{array}$ & $\begin{array}{c}\text { Matières } \\
\text { minérales } \\
(\%)\end{array}$ \\
\hline Décembre .. & 4,85 & 0,82 & Mai ........ & 4,75 & 0,89 \\
\hline Janvier ..... & 5,00 & 0,94 & Juin $\ldots \ldots \ldots$ & 4,37 & 0,93 \\
\hline Février ..... & 4,70 & 0,88 & Juillet ..... & 4,34 & 0,92 \\
\hline Mars ....... & 4,63 & 0,89 & Août ....... & 3,56 & 0,99 \\
\hline Avril ..... & 4,82 & 0,90 & Septembre... & 2,30 & 1,04 \\
\hline
\end{tabular}

\section{Utilisation du sérum}

Il a été procédé à beaucoup d'essais en ce qui concerne cette question. A l'exception du fromage de sérum, le sérum du lait de brebis n'offre pas de meilleures possibilités d'utilisation que celui du lait de vache. La pâte de sérum et la poudre de sérum sont plus riches en protéine que celles du lait de vache. La production du lactose présente plus de difficultés et est moins avantageuse en raison de la plus faible teneur en lactose et de la teneur plus grande en protéine et en sels minéraux résiduaires. Lors de nos essais, nous avons trouvé une teneur moyenne en extrait sec de $6,70 \%$ dans le sérum à la présure du lait de brebis, proportion 
s'élevant à $7,04 \%$ et redescendant à $6,06 \%$ à la fin de la période de lactation. Dans un cas, elle est tombée à $5,67 \%$.

Le colostrum du lait de brebis est d'un intérêt plutôt théorique. Le lait peut être considéré comme normal déjà quatre jours après agnelage ; mais les laiteries ont fixé un délai de huit jours avant que le lait ne puisse être livré. Le tableau suivant donne une idée de ce qu'est le lait colostral de 22 brebis; les écarts individuels ne sont considérables que pendant les deux premiers jours, et s'élèvent dans quelques cas à $30 \%$ des chiffres moyens indiqués.

TABLEAU XII

COLOSTRUM DU LAIT DE BREBIS

\begin{tabular}{|c|c|c|c|c|c|c|c|}
\hline & $\begin{array}{c}\text { Extrait } \\
\text { sec total } \\
(\%)\end{array}$ & $\begin{array}{c}\text { Matière } \\
\text { grasse } \\
(\%)\end{array}$ & $\begin{array}{c}\text { Protéine } \\
(\%)\end{array}$ & $\begin{array}{c}\text { Caséine } \\
(\%)\end{array}$ & $\begin{array}{l}\text { Lacto- } \\
\text { se }(\%)\end{array}$ & $\begin{array}{l}\text { Cen- } \\
\text { dres } \\
(\%)\end{array}$ & $\begin{array}{c}\text { Acidité } \\
\text { en acide } \\
\text { lactique } \\
(\%)\end{array}$ \\
\hline Premier lait ...... & 35,82 & 13,06 & 18,52 & 13,45 & 2,91 & 1,40 & 0,60 \\
\hline Après 1 jour . . . . . & 24,67 & 9,21 & 11,03 & 8,74 & 3,20 & 1,06 & 0,32 \\
\hline Après 2 jours .... & 21,39 & 9,02 & 8,01 & 5,34 & 3,11 & $1,01^{\circ}$ & 0,22 \\
\hline Après 3 jours .... & 19,58 & 7,71 & 7,97 & 5,01 & 3,02 & 0,84 & 0,19 \\
\hline Après 4 jours .... & 18,49 & 7,24 & 6,60 & 4,80 & 3,53 & 0,95 & 0,16 \\
\hline Après 5 jours .... & 18,04 & 6,71 & 5,91 & 4,92 & 4,41 & 0,92 & 0,16 \\
\hline Après 7 jours .... & 18,58 & 7,39 & 5,70 & 4,46 & 4,52 & 0,95 & 0,16 \\
\hline Après 9 jours ..... & 18,54 & 6,92 & 5,63 & 3,41 & 4,70 & 0,90 & 0,17 \\
\hline Après 1 mois $\ldots .$. & 18,23 & 6,93 & 5,42 & 4.08 & 4,72 & 0,95 & 0,19 \\
\hline
\end{tabular}

Teneur en chlore des laits de vache, de brebis et de chèvre

Comme il a été indiqué que la teneur en chlore de ces trois sortes de lait était très différente, ce problème a été examiné minutieusement. Actuellement, l'examen de la teneur en chlore se pratique journellement dans tous les laboratoires de laiterie et, avant tout, tout échantillon de lait suspect d'être fraudé n'est pas jugé sans une analyse de sa teneur en chlore. Les raisons de l'importance de ce test sont les suivantes : une teneur augmentée du lait de vache en chlore est fréquemment le premier symptôme d'une inflammation de la mamelle (mastite) et comme il arrive souvent que presque toutes les vaches d'une colonie sont infectées, les laiteries sont à même de mettre à la disposition du vétérinaire des produits pour les cas les plus urgents.

En outre, comme il a été indiqué que la teneur en chlore du lait de chèvre est bien plus élevée que celle du lait de vache ou du lait de brebis, la détermination de la teneur du lait en chlore a 
prouvé que c'était une bonne méthode pour déceler les additions: de lait de chèvre (cette méthode s'est montrée être bien plus exacte et bien plus facile d'exécution que la méthode de solubilité lente de la caséine de lait de chèvre dans l'ammoniaque).

Le tableau suivant indique les différences de la teneur en chlore des trois sortes de lait. Les chiffres résultent de la moyenne de plasieurs centaines de tests effectués sur chaque sorte de lait.

TABLEAU XIII

TENEUR EN CHLORE DES LAITS DE VACHE, BREBIS ET CHEVRE (MATIÉRE GRASSE PAR 100 GRAMMES)

\begin{tabular}{|c|c|c|c|}
\hline & Lait de vache & Lait de brebis & Lait de chèvre \\
\hline Moyenne ......... & 96,6 & 112,4 & 174,1 \\
\hline Minimum $\ldots \ldots \ldots \ldots$ & 66,2 & 96,0 & 142,0 \\
\hline Maximum $\ldots \ldots \ldots \ldots$ & 108,0 & 147,2 & 214,8 \\
\hline
\end{tabular}

Dans le lait des vaches souffrant d'une sérieuse inflammation des mamelles, la teneur en chlore peut s'élever à 164 mgr. sans changement de l'apparence extérieure du lait; toutefois, lorsque la teneur en chlore est plus élevée, on note une modification extérieure (grumeaux, couleur). Ci-dessous quelques exemples de laits de vaches souffrant de mastite sérieuse, lait dont l'aspect extérieur n'était pas modifié :

1. Matière grasse, $3,0 \%$ Poids spécifique, 1,0270

2. Matière grasse, $2,4 \%$

3. Matière grasse, $1,9 \%$

4. Matière grasse, $2,5 \%$
Poids spécifique, 1,0281

Poids spécifique, 1,0280

Poids spécifique, 1,0300
Extrait sec dégraissé, 7,49\% Extrait sec dégraissé, 7,62\% Extrait sec dégraissé, 7,52\% Extrait sec dégraissé, 8,14\%
Chlore, 164 mgr. 0 Chlore, $153 \mathrm{mgr}, 2$ Chlore, 152 mgr. 7 Chlore, $128 \mathrm{mgr}$. 7

Dans beaucoup d'analyses, le test du chlore nous a tout de suite suggéré que nous avions affaire à du lait non fraudé (addition d'eau) mais à du lait d'animaux atteints de maladies de la mamelle, observation toujours confirmée par les tests bactériologiques; d'où l'importance du test du chlore comme examen courant chaque fois qu'un lait nous apparaît suspect.

\section{Acidité titrable du lait de brebis}

La détermination du degré d'acidité ou "acidité titrable " est dans notre climat, et étant donné le manque de moyens de réfrigération, .une méthode indispensable pour l'élimination rapide à la plate-forme de réception des laits non absolument frais.

Déjà vers le début des années 1930 , le lait était payé d'après sa teneur en matière grasse, son degré d'acidité et sa propreté.

Une des raisons pour lesquelles les laiteries ne voulaient pas 
recevoir le lait de brebis était que son acidité titrable était souvent très élevée. L'acidité titrable est déterminée en Israël par le procédé Soxhlet-Henkel (S-H) : on opère sur $40 \mathrm{~cm}^{3}$ de lait après addition de phénolphtaléine avec de la $\mathrm{NaOHn} / 10$.

Dans ce qui suit, j'indiquerai entre parenthèses l'acidité titrable en acide lactique. Le $p \mathrm{H}$ du lait frais de brebis correspond à l'acidité titrable du lait de vache. En outre, il a été indiqué que le lait de brebis coagule lorsqu'on le chauffe au même degré que le lait de vache, c'est-à-dire à environ $12,0 \mathrm{~S}-\mathrm{H}=0,276 \%$ d'acide lactique.

Les plaintes sur la mauvaise qualité du lait de brebis continuèrent pendant de nombreuses années jusqu'à ce que des recherches minutieuses donnèrent les résultats suivants : l'acidité titrable élevée est une conséquence directe de la forte proportion de matières solides et est surtout produite par la caséine et les substances minérales. Elles ont prouvé que l'acidité est sujette à de grandes variations pendant la période de lactation et que la détermination de l'acidité titrable ne donne pas d'indication sur la fraîcheur, c'est-à-dire sur la qualité du lait.

Au début de la période de lactation, le lait de brebis a une acidité titrable équivalente à celle du lait de vache, et c'est là la principale raison de l'erreur. Il arrive que jusqu'en mai du lait d'animaux qui sont au début de la période de lactation est fourni en même temps que du lait d'animaux qui sont déjà au quatrième ou cinquième mois de la période de lactation. Ainsi une colonie peut fournir à la même heure un pot de lait frais qui est plutôt acide et un pot de lait parfaitement frais.

Le tableau suivant donne les résultats moyens des analyses de

TABLEAU XIV

AGIDITÉ TITRABLE DE LAIT DE BREBIS PENDANT LA SAISON

\begin{tabular}{|c|c|c|c|c|c|}
\hline & \multicolumn{3}{|c|}{ Tests à la laiterie } & \multicolumn{2}{|c|}{ Troupeaux sélectionnés } \\
\hline & \multirow{3}{*}{$\begin{array}{c}\text { S-H } \\
\% \\
6,85\end{array}$} & \multirow{2}{*}{\multicolumn{2}{|c|}{ Acide lactique }} & \multirow{2}{*}{$\begin{array}{c}\text { S-H } \\
\%\end{array}$} & Acide lactique \\
\hline & & & $\%$ & & $\%$ \\
\hline Décembre............. & & $=$ & 0,157 & 6,70 & 0,154 \\
\hline Janvier ................ & 8,10 & $=$ & 0,186 & 7,53 & 0,172 \\
\hline Février $\ldots \ldots \ldots \ldots \ldots \ldots$ & 8,32 & $=$ & 0,191 & 8,58 & $=0,197$ \\
\hline 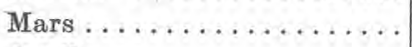 & 9,05 & $=$ & 0,208 & 9,14 & $=0,210$ \\
\hline Avril $\ldots \ldots \ldots \ldots \ldots$ & 9,12 & $=$ & 0,209 & 9,42 & $=0,216$ \\
\hline Mai $\ldots \ldots \ldots \ldots \ldots \ldots$ & 9,62 & $=$ & 0,221 & 9,85 & $=0,226$ \\
\hline Juin $\ldots \ldots \ldots \ldots \ldots \ldots$ & 9,48 & $=$ & 0,218 & 9,50 & $=0,219$ \\
\hline Juillet $\ldots \ldots \ldots \ldots \ldots \ldots$ & 9,62 & $=$ & 0,221 & 9,37 & $=0,215$ \\
\hline Août $\ldots \ldots \ldots \ldots \ldots \ldots$ & 8,82 & $=$ & 0,201 & 8,30 & $=0,191$ \\
\hline Septembre............. & 7,20 & $=$ & 0,161 & - & - \\
\hline
\end{tabular}


l'acidité titrable de laits de brebis livrés à des laiteries pendant une période de trois ans par deux troupeaux spéciaux de brebis.

Pour mieux illustrer comment l'acidité titrable dépend du stade de lactation, je donne ci-dessous les résultats de tests effectués sur un troupeau. Les brebis y agnelaient à des époques entièrement différentes (décembre à avril). Le lait frais d'un troupeau de 70 brebis avait une acidité titrable de 10,1 S-H $(0,232 \%)$, le lait d'un seul animal de $10,8(0,248 \%)$, le lait de 15 autres $9,2(0,212)$; et celui de 10 autres seulement $7,7(0,177)$ et celui de 3 animaux : 7,4 S-H $(0,172)$.

Bien entendu, toutes ces déterminations de l'acidité titrable ont été effectuées dans les mêmes conditions, c'est-à-dire sur du lait refroidi, deux heures après la traite.

En fait l'acidité titrable n'indique rien en ce qui concerne la frâेcheur du lait de brebis.

Comme les laiteries recevant le lait n'ont pas d'appareil indicateur de $p \mathrm{H}$ à leur disposition et qu'on ne peut pas se fier à la réaction colorimétrique (papier indicateur) avec le lait de brebis en raison de la forte viscosité de ce lait, il a fallu renoncer à l'acidité titrable et, par conséquent, aussi au paiement d'après l'acidité titrable.

Mais une autre question se posa : combien de temps le lait de brebis se conserve-t-il pour être en état d'être traité ? Logiquement, on peut dire que le lait ayant une acidité titrable de $10,0(0,23)$ atteindra plus rapidement l'acidité critique de $12,0 \quad(0,276)$ et se coagulera plus rapidement quand on le chauffera que le lait d'une acidité titrable de $7,0(0,16)$ et moins (étant entendu qu'ils sont à la même température, sont aussi propres et ont la même teneur en bactéries).

Au cours de nombreux tests et analyses, on a cherché à répondre à la question de l'augmentation de l'acidité titrable. Quelle est celle des trois sortes de lait qui se conserve le plus longtemps, malgré leur teneur différente en acide immédiatement après la traite? Les résultats ont été les suivants :

Le lait de brebis frais avec 50.000 bactéries par centimètre cube et une acidité de $9,6 \mathrm{~S}-\mathrm{H}(0,221)$ augmentera en acidité quand on le conservera à $20^{\circ}$, après 12 heures, et atteindra un degré d'acidité de $12,0 \mathrm{~S}-\mathrm{H}(0,276)$ après 22 heures.

Le lait de chèvre frais contenant le même nombre de bactéries et dans les mêmes conditions, mais d'un degré d'acidité de 6,2 S-H $(0,142)$ ne montre une première augmentation d'acidité qu'après 13 heures et atteint $12,0 \mathrm{~S}-\mathrm{H}(0,276)$ après 23 heures.

Le lait de vache frais d'une acidité de $6,7 \mathrm{~S}-\mathrm{H}(0,154)$ contenant. 
le même nombre de bactéries, conservé à la même température, ne montre une première augmentation d'acidité qu'après 12 heures et atteint un degré d'acidité de $12,0 \mathrm{~S}-\mathrm{H}(0,276)$ après 22 heures et demie.

De ce qui précède, nous voyons que, malgré une acidité titrable très différente au début, les diverses sortes de lait se conservent fraîches pendant à peu près le même laps de temps.

La formation d'acide lactique due à l'activité des bactéries apparaît beaucoup plus lentement dans le lait de brebis que dans les autres sortes de lait.

Au cours d'analyses bactériologiques spéciales, il a été trouvé que les bactéries se développent à la même vitesse dans les trois sortes de lait. On avait d'abord supposé que l'activité des bactéries dans le lait de brebis était plus lente, ce qui n'est donc pas le cas.

L'augmentation de l'acidité titrable a été comparée avec la détermination du $p H$ il n'a pas été déterminé d'écart considérable dans la diminution du $p \mathrm{H}$ entre les trois sortes de lait.

Un autre phénomène intéressant a encore besoin d'être étudié.

La diminution de l'acidité titrable dans le lait de vache dans les premières heures qui suivent la traite est un fait bien connu. Cette diminution est bien plus apparente dans le lait de brebis et atteint $1,2 \mathrm{~S}-\mathrm{H}(0,03)$, et même quelquefois plus dans quelques cas. Je désire mentionner ici que le même phénomène peut être observé, mais à un degré bien plus important, dans toutes les solutions de poudre de lait dans l'eau.

Le poids spécifique du lait de brebis, en Israël, est d'après mes observations, de 1,0371 et celui du lait de vache de 1,0316. En raison de la teneur très variable en matière grasse, le poids spécifique est sujet à de grandes variations : 1,0340-1,0396 et nous avons trouvé quelquefois de bien plus grandes variations dans les tests isolés.

Les formules habituelles pour la détermination des matières solides basées sur le poids spécifique et la teneur en matière grasse donnent, en ee qui concerne le lait de brebis, des résultats trop élevés. La formule :

$\mathrm{t}($ extrait sec dégraissé $)=\frac{\text { poids spécifique }}{4}+\frac{\text { matière grasse }}{5}-0,15$ nous rapprochera au plus près de l'extrait sec dégraissé (1).

Le point de congélation du lait de brebis est en moyenne de $-0,593$ et les variations maxima sont de -578 à $-0,613$.

(1) Pour un poids spécifique, par exemple de 1,0310 , on prend 31,0 et non 1,0310 . 
Le point de congélation moyen du lait de vache en Israël est de $-0,543$ variant de $-0,528$ à $-0,561$.

Le point de congélation moyen du lait de chèvre est de $-0,565$ variant de $-0,549$ à $-0,582$.

La détermination du point de congélation permet de voir si du lait de vache a été mélangé à du lait de brebis ; mais cette méthode ne donne rien avec des mélanges de lait de brebis et de lait de vache avee du lait de chèvre.

Constantes de la matière grasse dans le lait de brebis et le lait de chèvre. L'analyse des constantes exactes des diverses matières grasses de lait devint importante vers 1930, lorsqu'on soupçonna que le beurre local était fraudé. La fraude du beurre importé avec des graisses végétales n'était pas rare. Lors des premiers tests, on déclara que la matière grasse du lait de vache correspondait aux normes internationales. Les variations saisonnières étaient également faibles, l'indice d'iode (fourrage) variant le plus.

De grandes variations des chiffres furent notées de temps en temps dans les analyses de beurre provenant de laits mélangés de laiteries. On pensa d'abord que c'était la conséquence d'un mélange de lait de chèvre dont la littérature indique souvent que les constantes de sa matière grasse sont variables ; mais des tests systématiques nous indiquèrent, toutefois, que la matière grasse du lait de brebis est encore plus sujette à varier que la matière grasse du lait de chèvre ; mais, dans ce cas, nous avons aussi à noter la complication que la matière grasse varie suivant la saison et que, de temps en temps, la matière grasse du lait de brebis se différencie à peine de celle du lait de vache, alors qu'à d'autres moments elle s'en distingue considérablement.

Les valeurs limites des constantes des trois matières grasses de lait sont les suivantes.

A l'exception de l'indice de Polenske, le lait de chèvre est presque dans les limites de la matière grasse du lait de vache; mais ce qui est plus important pour révéler la fraude c'est que l'indice de Polenske de la matière grasse du lait de chèvre est toujours plus important. Dans le lait de brebis, toutes les constantes de la matière grasse sont plus élevées; mais à certains moments toutes ces constantes tombent au niveau de celles de la matière grasse du lait de vache.

A certains moments, la consistance du beurre provenant du lait de brebis ne peut pas être distinguée de celle du beurre de lait de vache alors qu'à d'autres moments elle en diffère considérablement. Ces différences peuvent être expliquées par le point de solidification très bas entre décembre et février et le point de fusion élevé en mai-juin. 


\section{TABLEAU XV}

CONSTANTES DE LA MATIËRE GRASSE DES MATIËRES GRASSES DE LAIT DE VAGHE, DE BREBIS ET DE GHËVRE (VALEURS MAXIMA ET MINIMA)

\begin{tabular}{|c|c|c|c|c|c|}
\hline \multirow{5}{*}{$\begin{array}{l}\text { Matière grasse du lait de vache ... } \\
\text { Matière grasse du lait de brebis ... } \\
\text { Matière grasse du lait de chèvre ... }\end{array}$} & \multirow{2}{*}{$\begin{array}{c}\begin{array}{c}\text { Indice de } \\
\text { Reichert }\end{array} \\
19,4-29,8\end{array}$} & \multicolumn{2}{|c|}{$\begin{array}{c}\text { Indice de } \\
\text { Polenske }\end{array}$} & \multirow{2}{*}{$\begin{array}{c}\begin{array}{c}\text { Indice } \\
\text { d'iode }\end{array} \\
27,8-38,2\end{array}$} & \multirow{2}{*}{$\begin{array}{c}\begin{array}{c}\text { Indice de } \\
\text { saponifica- } \\
\text { tion }\end{array} \\
217,3-239,2\end{array}$} \\
\hline & & \multicolumn{2}{|c|}{$1,2-2,9$} & & \\
\hline & $22,6-35,7$ & \multicolumn{2}{|c|}{$2,3-7,6$} & $29,3-38,8$ & $226,3-253,3$ \\
\hline & $\mid 19,9-29,2$ & \multicolumn{2}{|c|}{$4,4-7,4$} & $|30,1-38,6|$ & $230,1-235,7$ \\
\hline & \multicolumn{2}{|c|}{$\begin{array}{c}\text { Indice réfrac- } \\
\text { tométrique }\end{array}$} & \multicolumn{2}{|c|}{$\begin{array}{c}\text { Point de } \\
\text { solidification }\end{array}$} & $\begin{array}{l}\text { Point de } \\
\text { fusion }\end{array}$ \\
\hline Matière grasse du lait de vach $\Theta \ldots$ & \multicolumn{2}{|c|}{$40,2-46,3$} & \multicolumn{2}{|c|}{$18^{\circ} 7-25^{\circ} 1$} & $27 \circ 8-36 \circ 7$ \\
\hline Matière grasse du lait de brebis ... & \multicolumn{2}{|c|}{$41,2-49,4$} & \multicolumn{2}{|c|}{$14^{\circ} 4-20^{\circ} 6$} & $29^{\circ} 9-39^{\circ} 6$ \\
\hline Matière grasse du lait de chèvre... & \multicolumn{2}{|c|}{$41,6-44,2$} & \multicolumn{2}{|c|}{$19^{\circ} 3-24^{\circ} 8$} & $28^{\circ} 5-35^{\circ} 8$ \\
\hline
\end{tabular}

TABLEAU XVI

VARIATIONS DES CONSTANTES

DE LA MATIËRE GRASSE DU LAIT DE BREBIS PENDANT LA SAISON

\begin{tabular}{|c|c|c|c|c|c|c|c|}
\hline 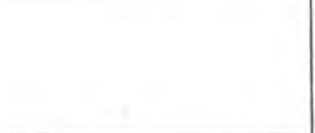 & $\begin{array}{c}\text { Indice } \\
\text { de } \\
\text { Reichert }\end{array}$ & $\begin{array}{l}\text { Indice } \\
\text { de Po- } \\
\text { lenske }\end{array}$ & $\begin{array}{l}\text { Indice } \\
\text { d'iode }\end{array}$ & $\begin{array}{c}\text { Indice } \\
\text { de } \\
\text { saconifi- } \\
\text { eation }\end{array}$ & $\begin{array}{c}\text { Indice } \\
\text { de } \\
\text { réfrac- } \\
\text { tion }\end{array}$ & $\begin{array}{c}\text { Point } \\
\text { de } \\
\text { solidi- } \\
\text { fication }\end{array}$ & $\begin{array}{l}\text { Point } \\
\text { de } \\
\text { fusion }\end{array}$ \\
\hline Décembre......... & 32,55 & 2,65 & 37,81 & 234,6 & 45,8 & $16^{\circ} 5$ & $31^{\circ} 8$ \\
\hline Janvier $\ldots \ldots \ldots \ldots$ & 32,83 & 3,20 & 36,30 & 240,2 & 43,2 & $15^{\circ} 7$ & $32^{\circ} 5$ \\
\hline Janvier $\ldots \ldots \ldots \ldots$ & 32,74 & 3,50 & 35,75 & 240,9 & 45,0 & $17^{\circ} 5$ & $33^{\circ} 4$ \\
\hline Février $\ldots \ldots \ldots \ldots$ & 33,67 & 5,22 & 33,62 & 245,0 & 43,2 & $14^{\circ} 5$ & $33^{\circ} 5$ \\
\hline Février $\ldots \ldots \ldots \ldots$ & 33,72 & 5,20 & 31,75 & 242,9 & 43,0 & $15^{\circ} 6$ & $30^{\circ} 5$ \\
\hline Mars $\ldots \ldots \ldots \ldots$ & 35,73 & 5,80 & 28,21 & 250,8 & 45,8 & $17^{\circ} 0$ & $31^{\circ} 2$ \\
\hline Mars ............ & 35,15 & 6,43 & 29,83 & 247,8 & 41,7 & $17^{\circ} 7$ & $30^{\circ} 0$ \\
\hline Avril $\ldots \ldots \ldots \ldots$ & 34,65 & 7,25 & 30,72 & 251,3 & 41,5 & $16^{\circ} 5$ & $32^{\circ} 0$ \\
\hline Avril $\ldots \ldots \ldots \ldots$ & 32,35 & 4,85 & 32,97 & 242,5 & 43,0 & $19^{\circ} 2$ & $35^{04}$ \\
\hline Mai ............ & 29,85 & 4,50 & 36,41 & 236,9 & 44,8 & $18^{\circ} 9$ & $36^{\circ} 7$ \\
\hline 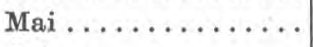 & 29,44 & 3,65 & 35,05 & 234,7 & 45,9 & $19^{\circ} 1$ & $37^{\circ} 1$ \\
\hline Juin $\ldots \ldots \ldots \ldots$ & 27,52 & 2,50 & 35,71 & 230,5 & 48,7 & $20^{\circ} 2$ & $39^{\circ} 4$ \\
\hline Juin $\ldots \ldots \ldots \ldots$ & 27,44 & 2,95 & 35,35 & 232,0 & 46,8 & $20^{\circ} 4$ & $38^{\circ} 3$ \\
\hline Juillet $\ldots \ldots \ldots \ldots$ & 27,20 & 2,70 & 32,34 & 235,2 & 46,0 & $18^{\circ} 6$ & $36^{\circ} 8$ \\
\hline Juillet $\ldots \ldots \ldots \ldots$ & 26,35 & 2,85 & 33,25 & 232,8 & 45,3 & $19^{\circ} 3$ & $36^{\circ} 6$ \\
\hline Août $\ldots \ldots \ldots \ldots$ & 25,42 & 2,70 & 35,10 & 232,2 & 47,5 & $20^{\circ} 2$ & $38^{\circ} 0$ \\
\hline Août $\ldots \ldots \ldots \ldots$ & 25,85 & 2,85 & 34,75 & 231,5 & 44,9 & $20^{\circ} 4$ & $38^{\circ} 2$ \\
\hline Septembre........ & 22,61 & 2,50 & 35,00 & 231,6 & 43,3 & - & \\
\hline
\end{tabular}


A mon avis, les assertions souvent très différentes exprimées dans la littérature sur la composition du lait de brebis, et qui sont très souvent absolument contradictoires, peuvent être expliquées par les variations saisonnières des constantes de la matière grasse de lait. Les tests, dont les résultats sont donnés ci-dessous, ont été effectués pendant de nombreuses années et les ehiffres indiqués sont des valeurs moyennes de nombreuses centaines d'analyses. Ce n'est qu'en décembre et en août que l'on a effectué le moins (15-20) d'analyses. Les chiffres ont été résumés en deux tableaux et un diagramme.

TABLEAU XVII

VARIATIONS DES CONSTANTES DE LA MATIËRE GRASSE DU LAIT DE GHËVRE

\begin{tabular}{|c|c|c|c|c|c|}
\hline & $\begin{array}{l}\text { Indice de } \\
\text { Reichert }\end{array}$ & $\begin{array}{l}\text { Indice de } \\
\text { Polenske }\end{array}$ & $\begin{array}{l}\text { Indice } \\
\text { d'iode }\end{array}$ & $\begin{array}{l}\text { Indice de } \\
\text { saponifica- } \\
\text { tion }\end{array}$ & $\begin{array}{l}\text { Indice } \\
\text { réfracto- } \\
\text { métrique }\end{array}$ \\
\hline Colostrum ....... & 19,9 & 2,6 & 38,5 & 216,5 & 45,8 \\
\hline Décembre ........ & 23,3 & 6,2 & 31,9 & 231,7 & 43,4 \\
\hline Janvier ......... & 28,2 & 6,1 & 34,6 & 233,1 & 42,0 \\
\hline Février . . . . . . . & 29,0 & 7,0 & 30,4 & 234,6 & 42,9 \\
\hline Mars . . . . . . . . . & 28,3 & 7,1 & 30,3 & 235,3 & 41,9 \\
\hline Avril $\ldots \ldots \ldots \ldots$ & 28,1 & 7,4 & 31,7 & 233,6 & 42,6 \\
\hline Mai ............. & 27,5 & 5,1 & 30,8 & 231,2 & 43,7 \\
\hline Juin . . . . . . . . & 28,4 & 5,6 & 31,8 & 230,8 & 42,5 \\
\hline Juillet $\ldots \ldots \ldots \ldots$ & 27,7 & 5,4 & 31,1 & 230,4 & 42,9 \\
\hline Août $\ldots \ldots \ldots \ldots$, & 27,1 & 5,3 & 32,4 & 230,9 & 42,9 \\
\hline Septembre ........ & 23,7 & 4,4 & 34,7 & 232,3 & 43,3 \\
\hline Octobre ........ & 22,1 & 4,9 & 31,6 & 235,5 & 41,8 \\
\hline
\end{tabular}

Les indices de Polenske, de Reichert et de saponification varient parallèlement d'une façon frappante, les indices d'iode variant en sens inverse.

Les variations les plus remarquables apparaissent dans les mois de mars à mai en même temps qu'une augmentation considérable de la teneur en matière grasse du lait.

Dans le lait de chèvre les variations des constantes de la matière grasse ne sont pas aussi manifestes que dans le lait de brebis; mais peuvent encore être observées dans les indices de Polenske et de Reichert. Ainsi qu'il l'a été été déjà mentionné une analyse de la matière grasse du lait de vache ne montre pas de variations nettes des constantes de la matière grasse.

Je ne désire pas m'étendre sur ces examens en émettant des théories sur les raisons de la variation des constantes, ou plutôt sur les variations des constituants de la matière grasse, les acides 


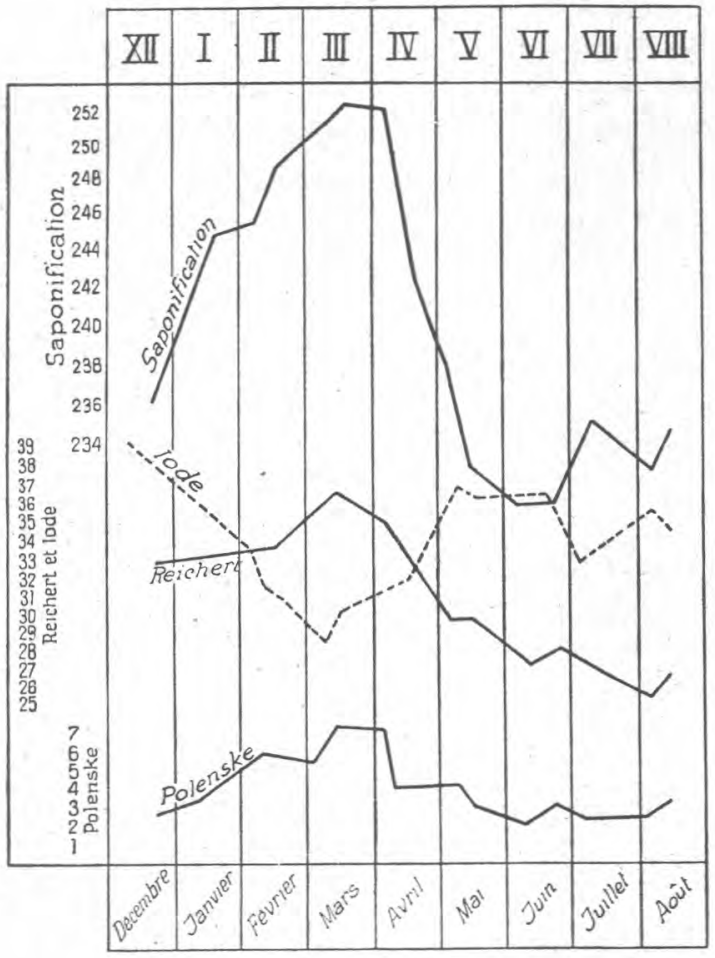

Diagramme :

Constantes de la matière grasse du lait de brebis

gras, pendant la période de lactation. Des analyses supplémentaires des acides gras isolés seraient nécessaires pour en donner une image plus nette.

Les analyses ont été d'une grande importance dans les problèmes de la fraude du beurre. Etant donné que des quantités considérables de matière grasse de beurre sont encore actuellement exportées des pays arabes et de l'Afrique orientale vers l'Europe pour la fabrication de beurre "rénové ", je crois qu'il est important d'attirer l'attention sur ces différences dans la composition des matières grasses. Tous ces pays ont des troupeaux importants de brebis et de chèvre et ne séparent pas toujours les diverses sortes de lait nécessaires à la production du beurre.

(Dans un second article sur l'Utilisation et le traitement du lait de brebis, il sera traité avec un peu plus de détail de la'question du Beurre de lait de brebis.) 